\title{
898.
}

\section{SUR L'ÉQUATION MODULAIRE POUR LA TRANSFORMATION DE L'ORDRE 11.}

[From the Comptes Rendus de l'Académie des Sciences de Paris, t. CXI. (JuilletDécembre, 1890), pp. 447--449.]

L'ÉQuation en $u, v$, en y écrivant $u=x, v=y$, est

$$
\begin{aligned}
& \begin{array}{rr}
y^{12} & \\
+y^{11} & \left(32 x^{11}-22 x^{3}\right)
\end{array} \\
& +y^{10} . \quad 44 x^{6} \\
& +y^{9} \quad\left(88 x^{9}+22 x\right) \\
& +y^{8} \cdot \quad 165 x^{4} \\
& +y^{7} \cdot 132 x^{7} \\
& \left.+y^{6}\left(-44 x^{10}+44 x^{2}\right)\right\}=0 \text {. } \\
& +y^{5}-132 x^{5} \\
& +y^{4}-165 x^{8} \\
& +y^{3}\left(-22 x^{11}-88 x^{3}\right) \\
& +y^{2}-44 x^{6} \\
& +y \quad\left(22 x^{9}-22 x\right) \\
& +1 .-x^{12}
\end{aligned}
$$

Selon un résultat trouvé par H. J. S. Smith, pour la transformation de l'ordre $p$, la courbe est de l'ordre $2 p$, et il y a à l'origine un point double, à l'infini deux points singuliers équivalents chacun à $\frac{1}{2}(p-1)(p-2)$ points doubles; et de plus $(p-1)(p-3)$ points doubles. Au cas $p=11$, le nombre de ces derniers points doubles est donc $=80$. Cela s'accorde avec l'expression

$$
D=x^{12}\left(1-x^{8}\right)^{10}\left(16 x^{16}-31 x^{5}+16\right)^{2}\left(x^{64}-301960 x^{56}+\ldots+1\right)^{2},
$$


trouvée par M. Hermite pour le discriminant de la fonction; et l'on voit ainsi que les valeurs de $x$, qui correspondent aux quatre-vingts points doubles, sont données par les équations

$$
\begin{array}{r}
16 x^{16}-31 x^{8}+16=0, \\
x^{64}-301960 x^{56}+\ldots+1=0 .
\end{array}
$$

Je ne considère que les seize points doubles donnés par la première équation. Cette équation donne

$$
x^{8}=\frac{1}{32}(31+3 i \sqrt{7}), \quad x^{4}=\frac{1}{8}(i+3 \sqrt{7}), \quad x^{2}=\frac{1+i}{4 \sqrt{2}}(-3+i \sqrt{7})
$$

il y a ainsi quatre points doubles, pour lesquels les valeurs de $x$ sont

$$
x= \pm \sqrt{\frac{1+i}{4 \sqrt{2}}}(-3+i \sqrt{7}), \quad x= \pm \sqrt{\frac{1+i}{4 \sqrt{2}}}(-3-i \sqrt{7})
$$

je trouve que les valeurs correspondantes de $y$ sont $y=\frac{1+i}{\sqrt{2}} x$, savoir que les quatre points sont situés sur la droite $y=\frac{1+i}{\sqrt{2}} x$, et, en changeant successivement les signes de $i$ et $\sqrt{2}$, on voit ainsi que les seize points sont situés, quatre à quatre, sur les droites

$$
y=\frac{1+i}{\sqrt{2}} x, \quad y=\frac{1-i}{\sqrt{2}} x, \quad y=-\frac{1+i}{\sqrt{2}} x, \quad y=-\frac{1-i}{\sqrt{2}} x
$$

J'écris, pour abréger,

done

$$
\begin{aligned}
& m=\frac{1+i}{\sqrt{2}},\left(\text { donc } m^{4}=-1\right) \\
& p=\frac{1}{4}(-3+i \sqrt{7})
\end{aligned}
$$

$$
2 p^{2}+3 p+2=0 \text {. }
$$

En écrivant $y=m x$ dans l'équation et en rejetant le facteur $x^{2}$, puis en écrivant $x^{2}=m p$, l'équation se présente sous la forme

$$
\left.\begin{array}{rl} 
& m^{10} p^{10} \cdot \quad 32 m^{11} \\
+ & m^{8} p^{8} \cdot \quad 88 m^{9} \\
+ & m^{7} p^{7} \cdot \quad 44 m^{10}-44 m^{6} \\
+ & m^{6} p^{6} \cdot-22 m^{11}+132 m^{7}-22 m^{3} \\
+ & m^{5} p^{5} \cdot \quad m^{12}+165 m^{8}-165 m^{4}-1 \\
+ & m^{4} p^{4} \cdot \quad 22 m^{9}-132 m^{5}+22 m \\
+ & m^{3} p^{3} \cdot \quad 44 m^{6}-44 m^{2} \\
+ & m^{2} p^{2} \cdot-88 m^{3} \\
+ & 1 \cdot-32 m
\end{array}\right\}=0
$$


où les coefficients ne contiennent que les puissances $m^{21}, m^{17}, m^{13}, m^{9}, m^{5}, m^{1}$ de $m$ et se réduisent ainsi à des multiples de $m$; il $\mathrm{y}$ a aussi un facteur numérique 8 , et, en divisant par $-8 m$, l'équation devient

$$
4 p^{10}-11 p^{8}-11 p^{7}+22 p^{6}+41 p^{5}+22 p^{4}-11 p^{3}-11 p^{2}+4=0
$$

cette équation est de la forme

$$
\left(2 p^{2}+3 p+2\right)^{2}\left(p^{6}-3 p^{5}+2 p^{4}+p^{3}+2 p^{2}-3 p+1\right)=0 .
$$

La droite $y=m x$ a donc, avec la courbe, quatre intersections doubles

c'est-à-dire

$$
p=\frac{1}{4}(-3 \pm i \sqrt{7})
$$

$$
x^{2}=\frac{1+i}{4 \sqrt{2}}(-3 \pm i \sqrt{7})
$$

on démontre sans peine que la droite n'est pas une tangente, et ces valeurs correspondent ainsi à des points doubles de la courbe, c'est-à-dire qu'il y a sur la droite $y=\frac{1+i}{\sqrt{2}} x$ quatre points doubles. Réciproquement, cette valeur de $x^{2}$ conduit au facteur $\left(16 x^{16}-31 x^{8}+16\right)^{2}$ du déterminant de l'équation modulaire. 